\title{
The ARM Southern Great Plains Central Facility Best Estimate Radiative Flux CD
}

April 2002

\author{
C. N. Long \\ Pacific Northwest National Laboratory \\ Richland, Washington
}

Work supported by the U.S. Department of Energy,

Office of Science, Office of Biological and Environmental Research 


\section{Contents}

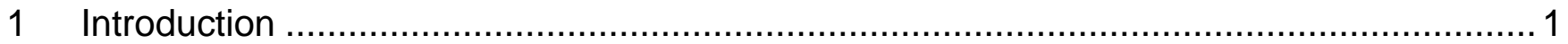

2 The Best Estimate Flux VAP (March 1997 - December 2001) ..................................... 1

3 Manual Determination of Best Estimate Flux (May 1995 - March 1997) ........................... 2

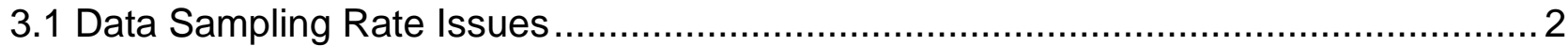

3.2 Data Quality Assessment Issues ....................................................................... 4

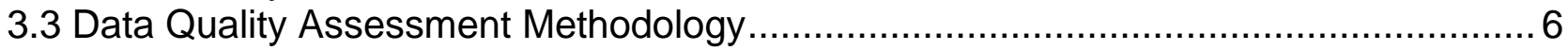

3.4 Determination of the Manual Best Estimate Flux .............................................. 11

4 Best Estimate Flux Data File Format and QC Flags ............................................... 12

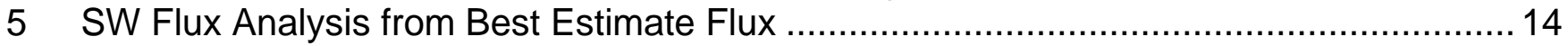

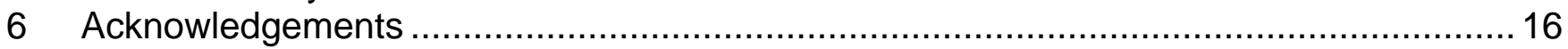

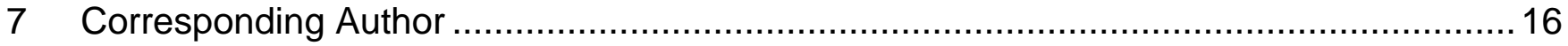

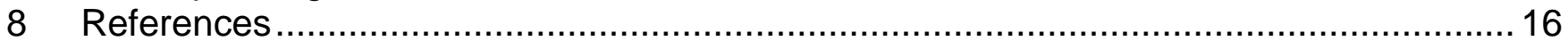

Figures

1 Cumulative frequency of differences between the SGP CF co-located SIROS and BRS radiometer data for the downwelling longwave (red), diffuse (blue) and direct normal (black) shortwave 1-minute averages.

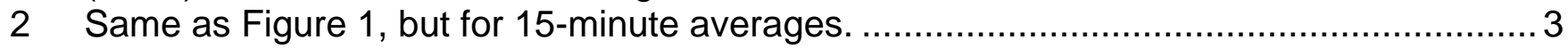

3 Same as Figure 2, but for clear-sky only ............................................................. 5

4 Comparison of ambient air temperature to corresponding brightness temperature calculated from downwelling longwave irradiance................................................... 7

5 Plot of all occurrences of positive cloud forcing greater than $30 \mathrm{Wm}^{-2}$ at the SGP CF in 2000 from 1-minute downwelling shortwave irradiance data ....................................... 8

6 Comparison of ambient air temperature to corresponding brightness temperature calculated from upwelling longwave irradiance

7 Frequency histogram of differences between ambient air temperature and corresponding brightness temperature calculated from downwelling longwave irradiance shown in Figure 6.

8 Comparison of corresponding upwelling and downwelling longwave irradiance from the SGP CF

\section{Table}

1 NetCDF and ASCII Data Labels for the Best Estimate Flux Files 


\section{Introduction}

The United States Department of Energy Atmospheric Radiation Measurement (ARM) Program operates a surface measurement site located near Lamont, Oklahoma. This Southern Great Plains (SGP) site is referred to in ARM as the SGP Central Facility (CF). ARM has operated three separate surface radiative energy budget measurement platforms at the CF since March 22, 1997, all located within a few tens of meters of each other. (Information on these measurement systems, called SIRS, SIROS, and BRS, is available through links on the ARM Web pages at http://www.arm.gov/ .) These surface radiation measurement platforms are operated under the guidelines and recommendations set forth by the Baseline Surface Radiation Network (Ohmura et al. 1998), and offer a unique opportunity for determining a best estimate of the surface radiative energy budget.

It is an axiom in data quality efforts that if one has only one measurement of a quantity, then quality assessment beyond testing against known physical limits is challenging. If one has two measurements of the same or very similar quantities, then one can both test against physical limits, and compare the two measurements. If both measurements are physically possible, but disagree beyond the expected accuracy of the measuring instruments, then it is often difficult to identify which is the "best" measurement. But if one has three or more measurements of the same or very similar quantities, and one measurement disagrees with the rest beyond the expected accuracy of the measuring instruments, then probability arguments can be used to infer that the "odd" measurement is most likely the "bad" one. This is the philosophical approach used in the Best Estimate Flux (BEFlux) Value Added Product (VAP) currently undergoing beta testing for ARM.

\section{The Best Estimate Flux VAP (March 1997 - December 2001)}

The BEFlux VAP directly compares data from the three Normal Incidence Perheliometers, shaded pyranometers, and shaded pyrgeometers at the SGP CF. Extensive analysis with several years of data has produced limits of typical ranges of agreement when these instruments are performing as expected. These limits are used to screen the data, and then the average is taken of the two that agree best, given that at least two instruments agree to within the established limits. This is done for the downwelling direct normal and diffuse shortwave, and the downwelling longwave. The total (global) downwelling shortwave is then the sum of the direct and diffuse components. Unfortunately, there are only two measurements each of the upwelling shortwave and longwave at the CF. We use only a comparison limit for the upwelling shortwave. For the upwelling longwave, we include comparisons with the co-located downward facing Infra-Red Thermometer. (More information on the BEFlux VAP analysis and testing techniques is presented in the ARM STM 2002 poster "Techniques and Methods used to determine the Best Estimate of Radiation Fluxes at SGP CF," and the corresponding extended abstract, by Shi and Long. This presentation includes estimates of what long-term field operational uncertainties actually are for these measurements, as opposed to accuracy estimates based only on highly controlled calibration activities as presented in Ohmura et al., 1998.)

In February of 2001, the ARM Program switched to using Eppley model 8-48 Black and White pyranometers for downwelling diffuse shortwave measurements. This change was prompted by study (Dutton et al. 2001) of the IR loss characteristics of single black detector pyranometers 
such as the Eppley model PSPs used by ARM previous to that date. It should be noted that the downwelling diffuse shortwave irradiance used in the Best Estimate products prior to the switch to Black and White diffuse pyranometers are the results from the ARM Diffuse Correction VAP. This VAP applies the correction technique suggested by Dutton et al. (2001) using information from co-located pyrgeometers and nighttime offset pyranometer data to empirically determine daytime corrections. (For more information of the Diffuse Correction VAP see Long et al. 2001; and the ARM STM 2002 poster "Results of the Dutton et al. IR Loss Correction VAP: Statistical Analysis of Corrected and Uncorrected SW Measurements," and the corresponding extended abstract, by Younkin and Long.)

\section{Manual Determination of Best Estimate Flux (May 1995 - March 1997)}

\subsection{Data Sampling Rate Issues}

Throughout the existence of the ARM Program, the operations, maintenance, calibration, and understanding of the surface irradiance measurements has constantly improved. Thus, surface irradiance data collected in 2001 is frankly of better quality than that collected in 1995. Prior to the addition of the SIRS C1 platform at the SGP CF in March of 1997, there were only two sets of radiometers operating. In addition, early in the program the broadband radiometer data were being collected with the co-located MFRSR data loggers. Because of the operational constraints of the MFRSR, the broadband data were only sampled once every 20 seconds, and are listed as SIROS data in the ARM Archive. At the CF, the SIROS E13 system was converted to the SIRS E13 system on August 25, 1997. The SIRS system uses a separate Campbell data logger that produces 1-minute averages from 1-second samples per BSRN recommended specifications. Fortunately, the longest operating set of radiometers at the CF, now called the BRS platform, has always produced 1-minute averages from 1-second samples.

The consequences of using only 20-second sampling were reported by Long (1996) shortly after the first ARESE. In comparing the 1-second sampled data to the corresponding 20-second sampled data, differences on the time scale of 1-minute are shown to be very large under cloudy skies, especially for the direct normal shortwave irradiance measurements. Long (1996) recommended that SIROS data be averaged for at least 15 to 30 minutes to help overcome the SIROS under sampling. Figures 1 and 2 help illustrate this point by showing the cumulative frequency of differences between the 1-second and 20-second sampled data for 1-minute (Figure) and 15-minute (Figure 2) averages. For the 1-minute data, 95\% of the measurements of downwelling longwave agree to within about $6 \mathrm{Wm}^{-2}$. For the diffuse shortwave, 95\% of the data agree to within about $20 \mathrm{Wm}^{-2}$. Both these measurements are relatively slowly changing quantities compared to the direct normal shortwave, primarily because the direct normal is taken from such a small area of the sky (a $5^{\circ}$ field-of-view) of a potentially rapidly varying and large quantity. Thus in this case, Figure 1 shows that the 1-minute direct normal shortwave measurements do not reach the $95 \%$ level within the $50 \mathrm{Wm}^{-2}$ range of the plot.

For the 15-minute average comparison shown in Figure 2, again 95\% of the measurements of downwelling longwave agree to within about $6 \mathrm{Wm}^{-2}$, evidence of the slowly changing nature of the field. For the diffuse shortwave, 95\% of the data now agree to within about $14 \mathrm{Wm}^{-2}$. But the most dramatic improvement in agreement is shown for the direct normal shortwave, where $95 \%$ of the 15 -minute data now agree to within about $28 \mathrm{Wm}^{-2}$. 
Daylight All-Sky Cumulative Frequency of 2 Radiometer Differences

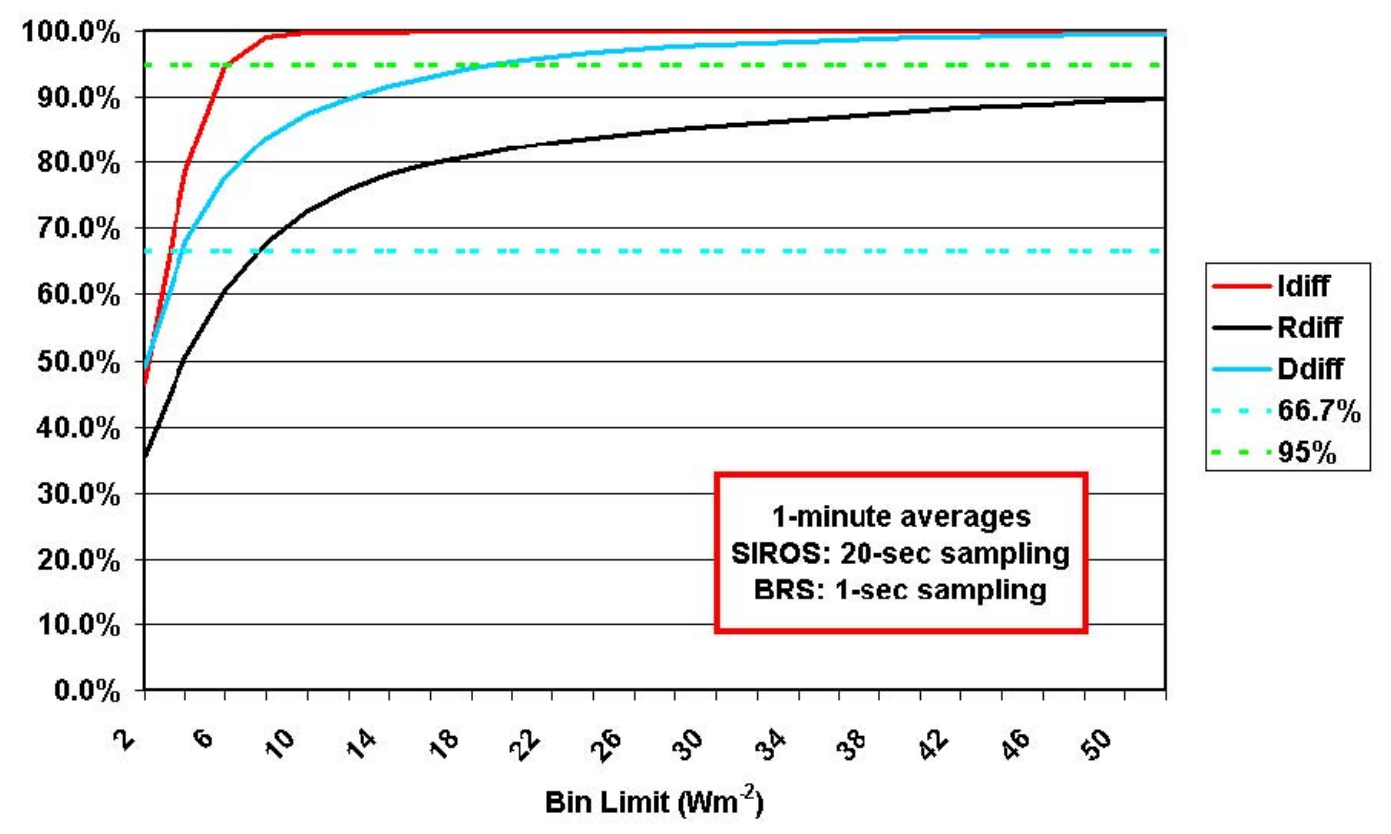

Figure 1. Cumulative frequency of differences between the SGP CF co-located SIROS and BRS radiometer data for the downwelling longwave (red), diffuse (blue) and direct normal (black) shortwave 1-minute averages. Data span from 19950928 through 19970824.

Daylight All-Sky Cumulative Frequency of 2 Radiometer Differences

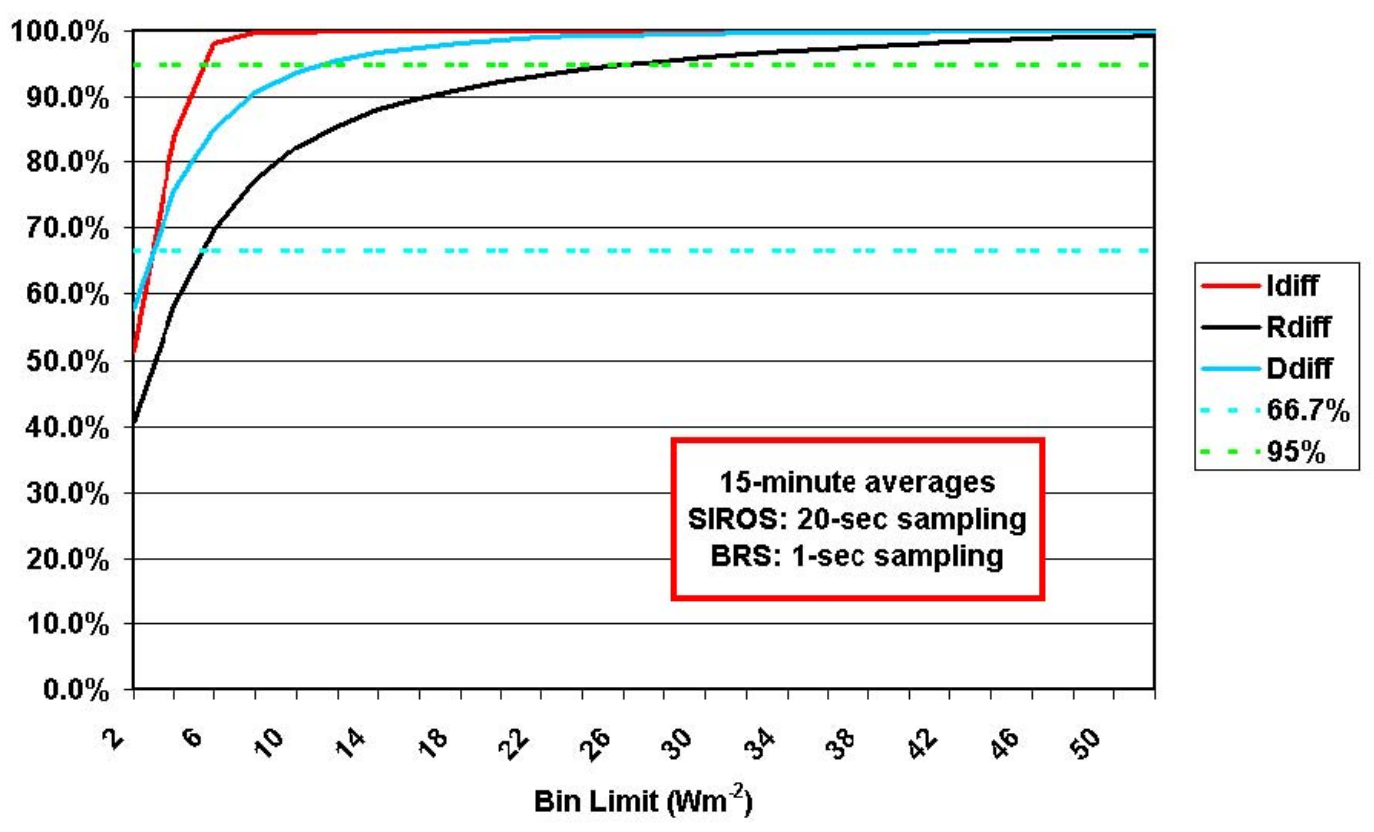

Figure 2. Same as Figure 1, but for 15-minute averages. 
The measurement differences shown in Figures 1 and 2 are primarily due to two related factors, both of which are ameliorated by averaging the data. The first factor relates to the sampling rate. The lower the sampling rate for changing fields, the less representative short duration averages are of the variability inherent in the field. Thus, one must take longer time averages to the point wherein the average is based on a statistically representative set of samples. In addition, and related, small time offsets between the two co-located logging systems can also produce large differences for narrow field-of-view quantities. This is not as large a factor for those measurements representing a larger, in this case hemispheric, field-of-view. Since the field being measured is slowly evolving compared to the averaging, a small time offset such as 5 seconds will still contain the variation in the field-of-view. But the same 5-second timing difference for the $5^{\circ}$ field-of-view of the direct normal instrument means the variation in the field has often completely moved out of the field-of-view, and the sub-sampling co-located instrument has now sampled a completely different situation. Again, the data must be averaged to the point where the average is statistically representative, but the narrower the field-of-view, the longer the averaging time needed.

One situation where the difference in sampling is not a factor is for the very slowly changing situation of clear skies. Figure 3 shows the cumulative frequency similar to Figures 1 and 2, but now screened for only clear-sky cases using the technique of Long and Ackerman (2000). Here, the sampling strategy is unimportant. The agreement shown in Figure 3 reflects the effects of factors inherent in operating these instruments in the field, imbedded in the vagaries of the ambient conditions and subject to such things as rain, deposition on the radiometer domes, and the like. For the clear-sky data, 95\% of the measurements of downwelling longwave agree to within about $5 \mathrm{Wm}^{-2}$, not much different from the results in Figures 1 and 2 and again testifying to the slowly changing nature of the field. For the diffuse shortwave, $95 \%$ of the data agree to within about $8 \mathrm{Wm}^{-2}, 6 \mathrm{Wm}^{-2}$ better agreement than for 15-minute averages of all-sky data. But as expected, the most dramatic improvement in agreement is shown for the direct normal shortwave, where $95 \%$ of the 15 -minute data agree to within about $12 \mathrm{Wm}^{-2}$.

If the Best Estimate Flux data is made available at high temporal resolution, in this case 1-minute data, then those desiring longer time averages can easily produce them. But the converse is not true. Because of the sampling differences between the SIROS and BRS systems and the desire to produce a dataset that satisfies both short and longer time average requirements, I have chosen to preferentially use the 1-second sample data in producing the best estimate, all else being equal. The "all else being equal" is primarily related to a fundamental assessment of data quality.

\subsection{Data Quality Assessment Issues}

In the case of the Best Estimate Flux VAP, data quality assurance is inherent in the comparison of three measurements of the same quantity for these systems that are routinely monitored and maintained on a daily basis. This is especially true given the agreement limits that have resulted from our long-term analysis during development. For the manually produced Best Estimate, we only have two measurements to work with, and thus quality assurance becomes a more difficult task. Fortunately we have tools to bring to bear that include past records and work by colleagues, and our own development work associated with other VAPs. 
Daylight Clear-Sky Cumulative Frequency of 2 Radiometer Differences

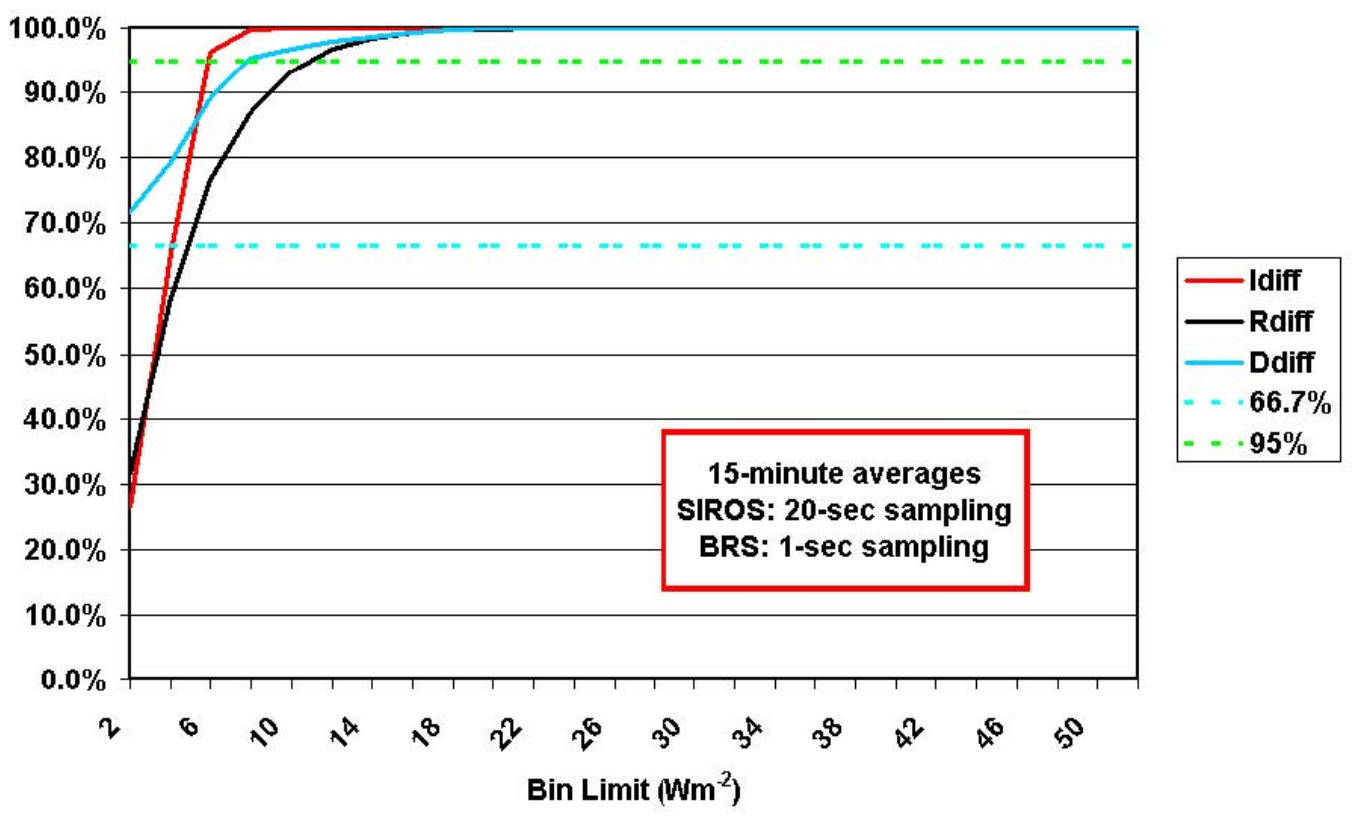

Figure 3. Same as Figure 2, but for clear-sky only.

As with any long-term measurement undertaking, there is bound to on occasion be missing data. This is not unusual, and often happens for brief periods under normal operation, such as the once per year calibration instrument swap-outs. However, other times equipment problems can result in extended periods of either missing or known poor quality and unreliable data. An example is the case with the E13 SIROS system prior to September 23, 1995. The E13 MFRSR logger experienced line transformer problems that make the data before this date unusable (Ted Cress and Robin Perez, personal communication). These data are no longer available through the ARM Archive. Known periods such as this have been removed from the Manual Best Estimate time series, according to information supplied by Marv Wesely and Tom Stoffel.

One known problem has been addressed shortly after the first ARESE. Partly through the reports of Long (1996) and others, an on-site comparison was made with the two radiometer systems and cavity radiometers. The following is an excerpt from documentation provided by Marv Wesely:

“A comparison of BSRN and SIROS solar radiometers for measuring downwelling irradiances at the SGP central facility was made with field standards and two absolute cavity radiometers brought to the site or a two-week period in April 1996 by Mike Rubes (formerly of the National and Oceanic Atmospheric Administration, Air Resources Laboratory, Surface Radiation Research Branch in Boulder, Colorado). A description of this effort can currently be found on the World Wide Web at http://www.srrb.noaa.gov/ apr96iop/hagsie.html . Analyses of the data from these comparisons have resulted in several observations on the quality of data collected at the BRS and SIROS platforms since October 13, 1995, which are probably valid to the present time, until these sensors are replaced with more recently calibrated sensors. On October 13, 1995, the two BRS pyranometers (PSPs) were replaced, so the observations do not apply to the BRS measurements of global and diffuse irradiation before that date. Another source of 
information is inspection of the SIROS and BRS equipment by Joe Michalsky (Atmospheric Science Research Center, State University of New York at Albany) at various times. The results of the findings are summarized as recommendations in the following several paragraphs. Some explanation and further comments are provided in the parenthetical remarks.”

A brief summary includes the discovery that the BRS direct normal shortwave was overestimated by about 0.5\%, while the SIROS direct normal was underestimated by about 2.1\%. To compensate for this, it was recommended that the BRS direct normal be multiplied by a factor of 0.995, and the SIROS by 1.021. (Per Marv Wesely documentation: The analyses leading to these recommendations are described in an extended abstract presented in early February: J. Michalsky et al. "Optimal Measurements of Surface Shortwave Irradiance Using Current Instrumentation-The ARM Experience,” in Preprint Volume, Ninth Conference on Atmospheric Radiation, February 2-7, Long Beach, California, pp. J5-J9, American Meteorological Society, Boston, Massachusetts) (See also Michalsky et al. 1999). These recommended factors have been applied to the direct normal data for the appropriate time periods. In fact, the direct normal data used to produce Figures 1-3 were adjusted in this manner.

\subsection{Data Quality Assessment Methodology}

Each data stream is first analyzed for data quality. As previously mentioned, the diffuse shortwave used in the manual Best Estimate were produced by the ARM Diffuse Correction VAP to correct for IR loss. Analyses results (Long et al. 2001; Younkin and Long 2002) suggest that perhaps the full correction, using both the pyrgeometer detector flux and the pyrgeometer case and dome temperature information, produces on average the better correction. However, the full correction requires more pieces of information, which is not always reliable. When it is determined that the needed information might not be reliable, no full correction is made to the diffuse shortwave data. Thus, the detector-only diffuse shortwave correction, being simpler, is more often available. To account for this, the full correction diffuse shortwave is used if available for each data stream, and if not then the detector-only corrected diffuse is used. In addition, the adjustments to the direct normal shortwave were applied where appropriate.

Each data stream is then checked for reasonable physical limits. In the case of the downwelling longwave irradiance, the Stephan-Boltzman relation is used to calculate the equivalent brightness temperature. This brightness temperature is then compared to the corresponding air temperature measurement. It is intuitively obvious that the brightness temperature calculated from downwelling longwave irradiance should be no greater than the ambient air temperature. Figure 4 shows about 1 year of data from the SGP CF comparing the ambient air temperature to the corresponding downwelling longwave brightness temperature. As the plot shows, the majority of the time the downwelling brightness temperature is less than the air temperature. The few times the two are about equal occur under foggy or hazy conditions. Of course, both the pyrgeometer and the thermometer measuring air temperature have uncertainties associated with them. Thus, this physical limit test allows the downwelling longwave brightness temperature to be less than the air temperature plus $1 \mathrm{~K}$ to account for the uncertainties. The downwelling longwave must also be a positive value. 
Comparison of Ambient Air and IRdn Brightness Temperatures, SGP CF

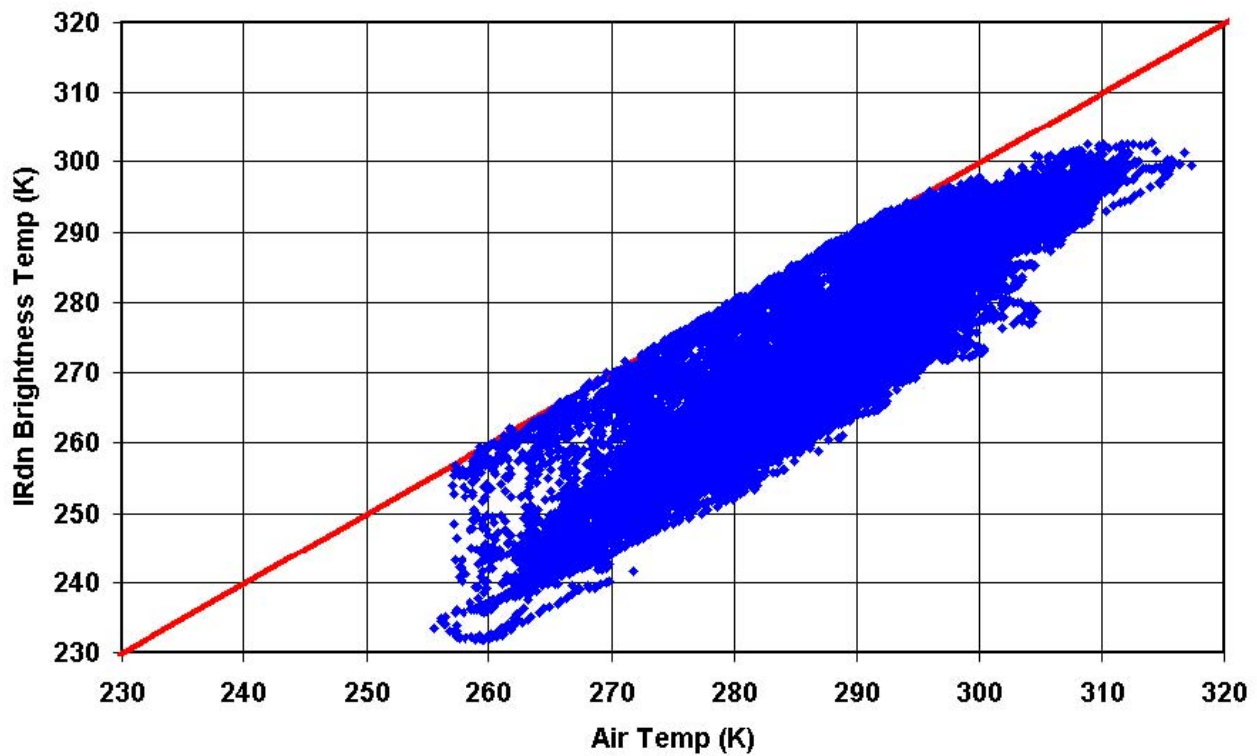

Figure 4. Comparison of ambient air temperature to corresponding brightness temperature calculated from downwelling longwave irradiance. Red line represents $\mathrm{X}=\mathrm{Y}$.

The direct normal shortwave irradiance is tested for a reasonable range of values. The normal incidence perheliometer is also subject to some small IR loss, thus can produce slightly negative values. The maximum values should be no larger than the solar constant, attenuated by a typical amount of clear-sky atmospheric scattering and absorption. Thus, the direct normal irradiance limits are set to a minimum of $-20 \mathrm{Wm}^{-2}$ and a maximum of $1200 \mathrm{Wm}^{-2}$ for the data to be accepted as possible.

The downwelling diffuse and total (global) shortwave are tested for reasonable ranges. Again, the minimum is set to a value of $-20.0 \mathrm{Wm}^{-2}$. The pyranometers used for these measurements are flat plate detectors, meaning that the detector surface is perpendicular to zenith. Thus, the effect of the incident irradiance is weighted by the cosine of the angle from zenith. The total shortwave measurement includes both the direct and diffuse components. In addition, these "point" measurements are subject to periods of irradiance values that are greater than the corresponding clear-sky amounts due to the presence of clouds, a phenomenon known as positive cloud forcing. To account for these behaviors, a limit based on a function with the cosine of the solar zenith angle as the independent variable is used. As reported in Long and Ackerman (2000), a simple power law function works well for capturing the clear-sky total shortwave diurnal cycle. Figure 5 shows a plot by cosine of the solar zenith angle of 1 year of 1 -minute data from the SGP CF when the measured total shortwave exceeded the corresponding clear-sky amount by at least $30 \mathrm{Wm}^{-2}$. The plot also shows a power law function (red) where the multiplicative constant is set to $1500 \mathrm{Wm}^{-2}$, the exponential coefficient is set to 1.2 (Long and Ackerman 2000), and an extra $50 \mathrm{Wm}^{-2}$ is added as an offset. Note that only 81 -minute measurements of total downwelling shortwave from unshaded pyranometer measurements fall above this function. The total shortwave measurements from the unshaded pyranometer are used for comparison testing, since the final Manual Best Estimate total shortwave value is calculated 


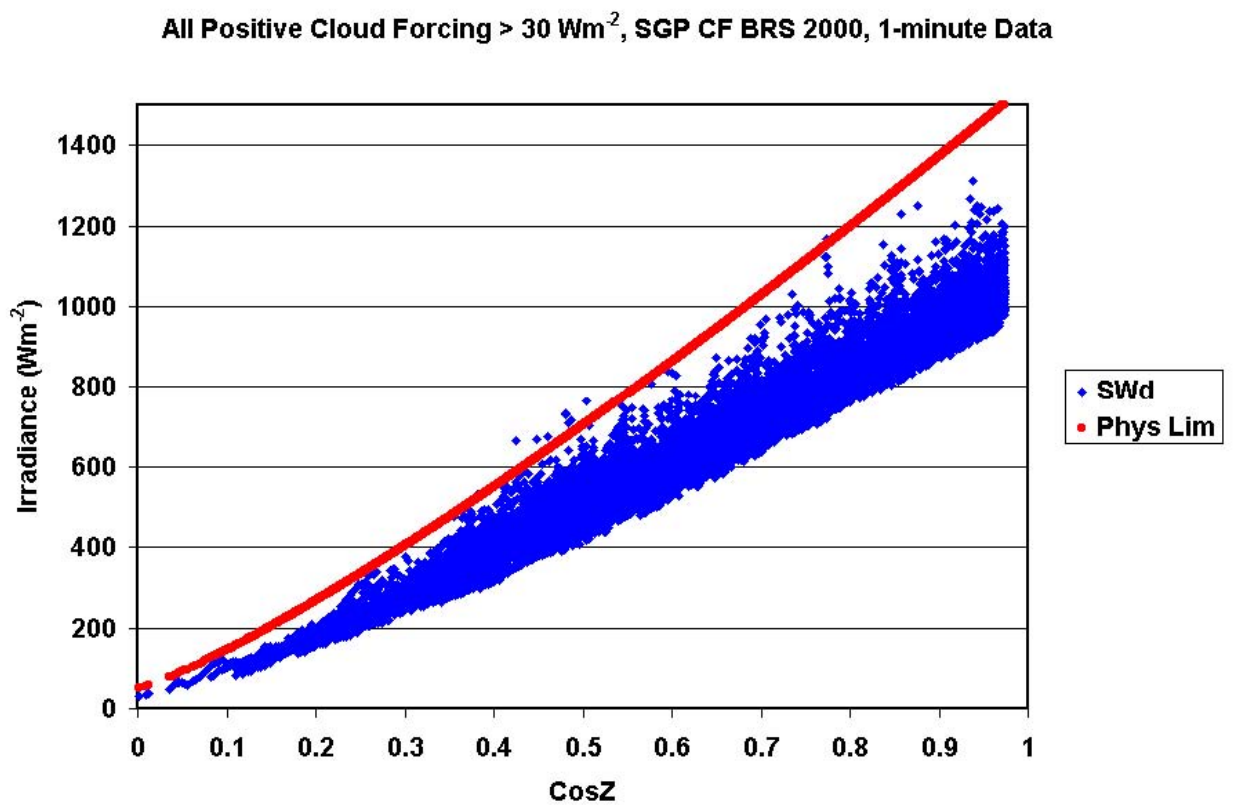

Figure 5. Plot of all occurrences of positive cloud forcing greater than $30 \mathrm{Wm}-2$ at the SGP CF in 2000 from 1-minute downwelling shortwave irradiance data. Red line represents the function 1500 Wm-2 (CosZ) $1.2+50$ Wm-2.

as the sum of the best direct and diffuse components. Thus, it is acceptable to "lose" these few data in order to better screen for unreasonable data. Both the total (from the unshaded pyranometer) and diffuse downwelling shortwave are compared to this maximum limit.

Once the direct normal, total, and diffuse shortwave are tested against the above minimum and maximum limits, the unshaded pyranometer total shortwave is compared to the sum of the direct and diffuse shortwave components. (The direct component is calculated as the direct normal shortwave times the cosine of the solar zenith angle.) If the total shortwave is greater than $100 \mathrm{Wm}^{-2}$, then "good" component sum data is expected to fall within $25 \%$ of the total shortwave, or $100 \mathrm{Wm}^{-2}$, whichever is the smaller number. If the total shortwave is less than $100 \mathrm{Wm}^{-2}$, then the "good" component sum is expected to agree within $25 \mathrm{Wm}^{-2}$. (This may seem a rather large allowance, but here we are screening for fairly obvious problems, and do not want to eliminate "good" data by too tight a restriction. Other tests will further refine the quality assessment. In addition, as shown by Michalsky et al. 1999, the cosine response errors of the unshaded pyranometer alone can at times produce errors of $30 \mathrm{Wm}^{-2}$ or more. This is why BSRN recommends the component sum as the better measurement.) For data that do not pass these tests, both the direct and diffuse shortwave are labeled as "bad," since we have no further information as to which is actually problematic.

The only upwelling shortwave and longwave irradiance measurements during the period of the Manual Best Estimate come from the E13 system, when available. We test these values using some of the previously tested downwelling values. Since the upwelling shortwave is that portion of the downwelling that is reflected from the surface, the upwelling shortwave should not be greater than the downwelling, even for highly reflective surface conditions such as snow cover. If the downwelling shortwave is greater than $10 \mathrm{Wm}^{-2}$, and the upwelling shortwave is less than 
this amount, then it is deemed acceptable. Because of the pyranometer uncertainties of these measurements, if the downwelling shortwave is less than $10 \mathrm{Wm}^{-2}$ and the absolute difference between the upwelling and downwelling shortwave is less than $10 \mathrm{Wm}^{-2}$, then the data are accepted.

To test the upwelling longwave, we again calculate a brightness temperature and compare it to the corresponding ambient air temperature. Figure 6 shows a plot similar to Figure 4, but with the upwelling longwave brightness temperature on the Y-axis. In this case, the brightness temperature can be either greater than or less than the corresponding air temperature, though not greatly so. While the air temperature near the surface is primarily determined through such mechanisms as convection and winds, radiative processes often dominate the radiating surface of the ground. Thus, strong shortwave energy deposition often heats the surface to higher temperatures than the air above, while longwave emission at night often cools the surface to lower temperatures than the air above. Nevertheless, the temperature differences are not drastic at the SGP CF. Figure 7 shows a frequency histogram of the differences between the corresponding air and surface brightness temperatures shown in

Figure 6. As Figure 7 shows, the range of temperature differences is small, spanning only across about $12 \mathrm{~K}$ either way. Thus, to test for reasonable upwelling longwave measurements, we require that the calculated brightness temperature be within $13 \mathrm{~K}$ of the ambient air temperature, to allow for air temperature measurement uncertainties.

In addition, the upwelling longwave irradiance itself is compared to the corresponding downwelling longwave irradiance measurement. Figure 8 shows a comparison of corresponding upwelling and downwelling longwave irradiance from the SGP CF. As stands to reason; most often the upwelling longwave is greater than the downwelling longwave. The times when they are about equal are again associated most often with fog and haze events. Thus, the upwelling longwave is also tested against the downwelling longwave, and deemed acceptable if the downwelling is less than the upwelling plus a factor of $10 \mathrm{Wm}^{-2}$ to account for pyrgeometer uncertainties.

One means of detecting some more subtle shortwave measurement problems is included in the algorithm of the ARM Shortwave Flux Analysis VAP. This algorithm applies two tests using the ratio of measured over clear-sky downwelling shortwave, and the ratio of measured diffuse over measured total shortwave. These tests detect when the solar tracker was off alignment, or there was an unknown subtle problem with one of the three instruments: the unshaded and shaded pyranometers, and the normal incidence perheliometer. (These tests are described in Long 2001.) In order to use these tests, the estimated clear-sky shortwave is needed for each system. Each of the diffuse corrected data sets was processed through the Long and Ackerman algorithm to determine the appropriate daily clear-sky fit coefficients, and time series of clear-sky irradiance estimates. As described in Long (2001), if the measured over clear-sky total shortwave ratio is greater than 0.9 , while the corresponding ratio of measured diffuse over total shortwave is also greater than 0.9 , then this is an indication that the solar tracker was not properly aligned to track the sun. If this was the case, the component data are marked as "bad." Similarly, the measured over clear-sky total shortwave ratio calculated using the unshaded 
Comparison of Ambient Air and IRup Brightness Temperatures, SGP CF

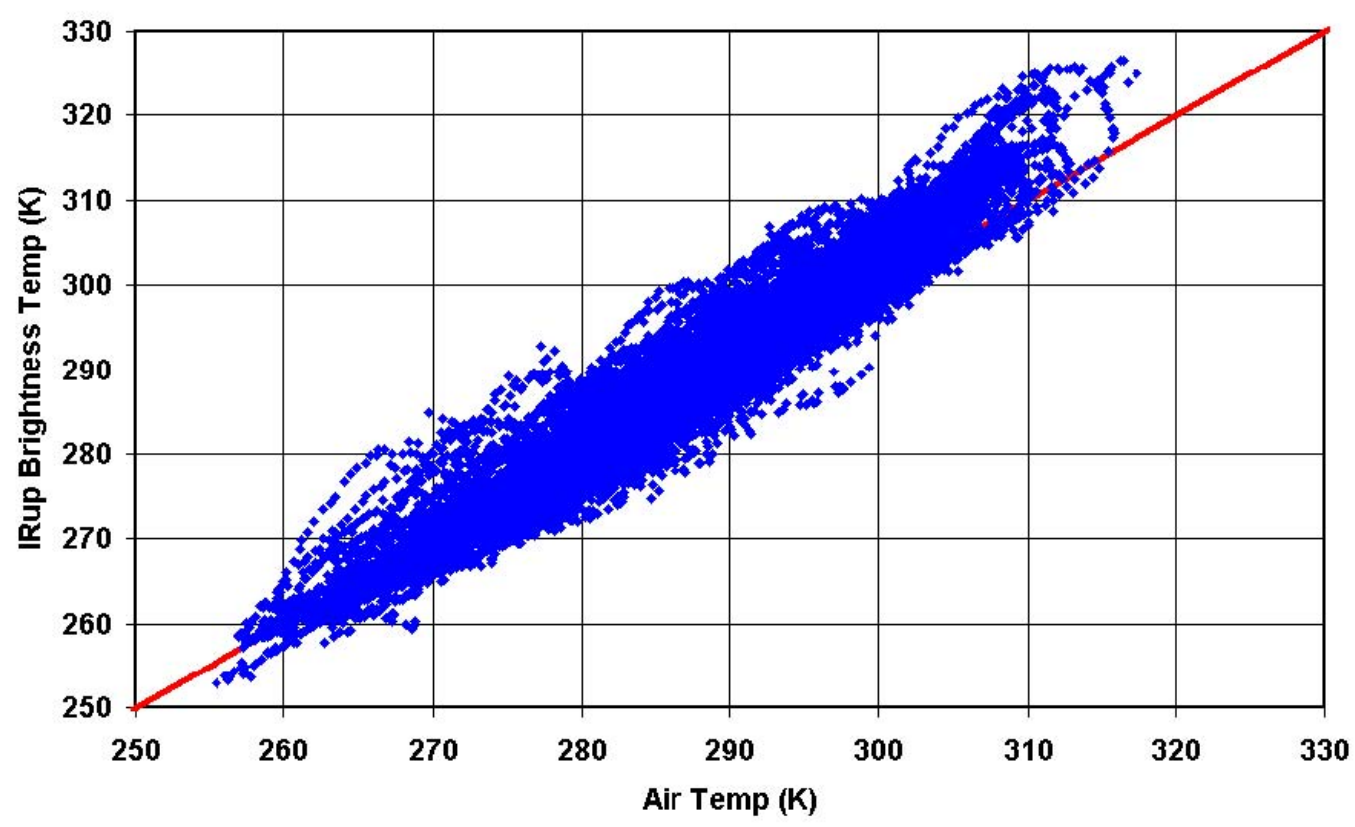

Figure 6. Comparison of ambient air temperature to corresponding brightness temperature calculated from upwelling longwave irradiance. Red line represents $\mathrm{X}=\mathrm{Y}$.

Frequency of Difference, Air Temperature Vs. IRup Brightness Temperature

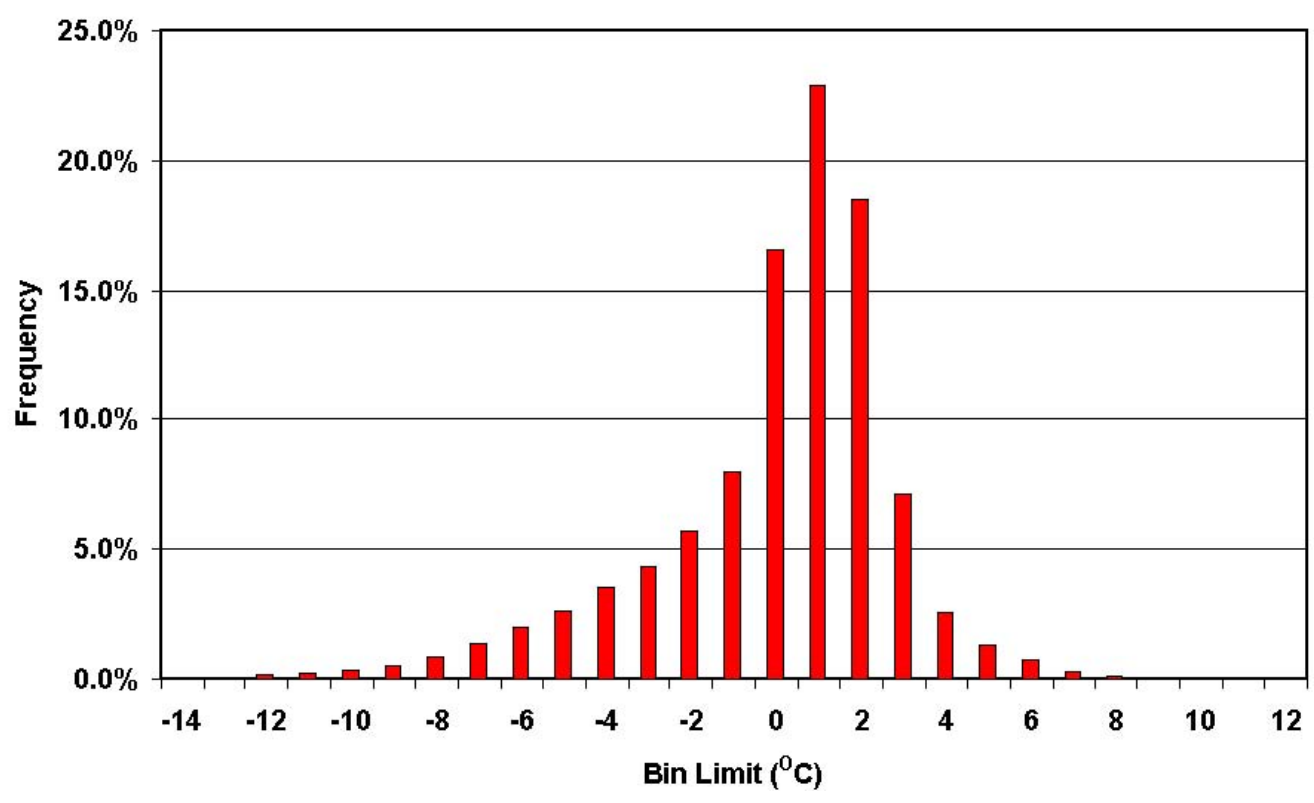

Figure 7. Frequency histogram of differences between ambient air temperature and corresponding brightness temperature calculated from downwelling longwave irradiance shown in Figure 6. 
Comparison of Upwelling and Downwelling Longwave, SGP CF

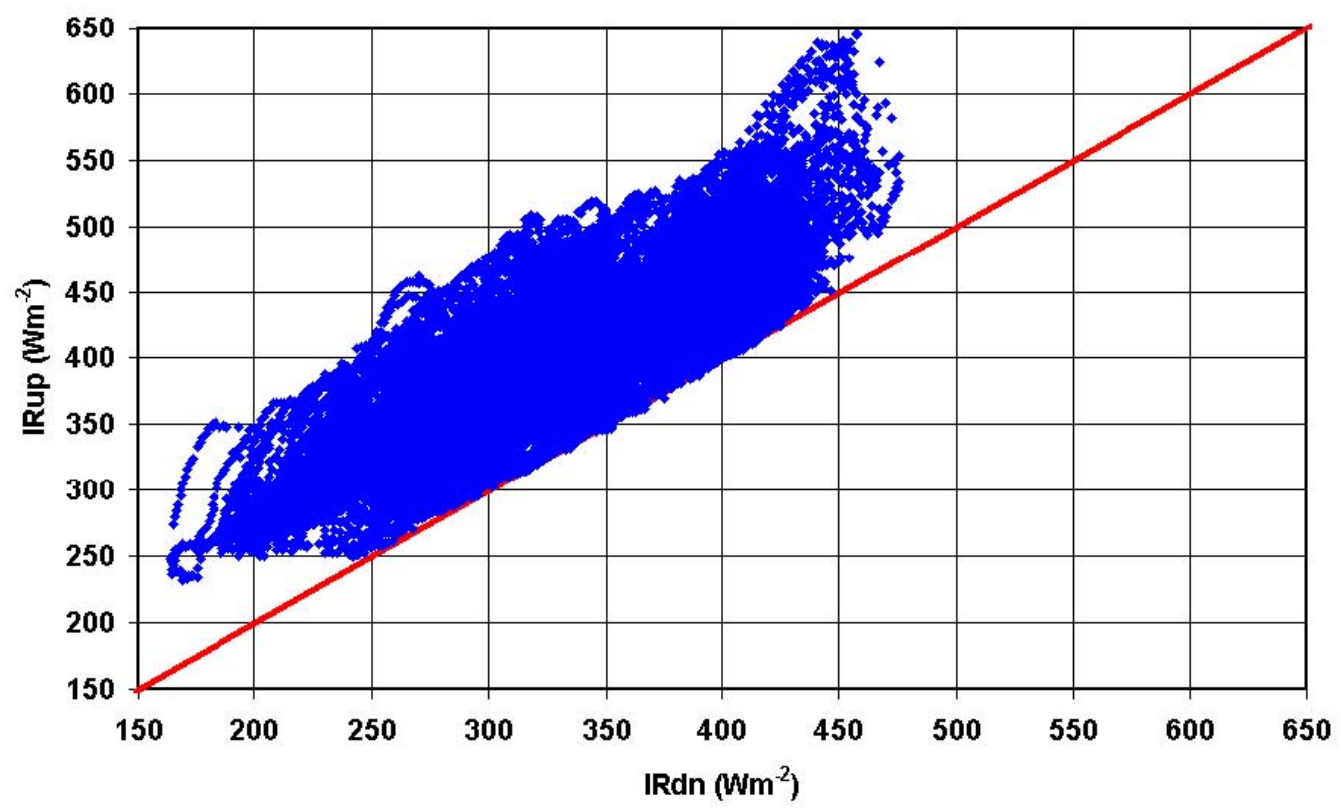

Figure 8. Comparison of corresponding upwelling and downwelling longwave irradiance from the SGP CF. Red line represents $X=Y$.

pyranometer data is compared to the same ratio calculated using the component sum data. Under normal circumstances, these two ratios are generally almost identical to each other in value. If these two ratios differ by more than $8 \%$, it is an indication that one of the three shortwave instruments has a problem, and again the component data is marked as "bad."

Once all of the preceding data quality assessment is performed, the remaining data are about as well screened as possible given the available information. These screened data from each of the two systems are then compared to determine the Best Estimate.

\subsection{Determination of the Manual Best Estimate Flux}

The quality-tested data were combined to produce the Manual Best Estimate Flux using the information presented in the preceding sections of this document.

For the upwelling irradiances, as stated before, there is only one set of measurements available. Those upwelling measurements that pass all data quality tests are then, by definition, the best available and are included in the output.

For the direct normal shortwave irradiance, using the results presented in Figures 1-3, if the 1 -second data is available it is used, else the 20-second sampled data is used.

For the diffuse shortwave irradiance, we use the 95\% cumulative frequency results from Figure 3 as a limit. The actual calibration accuracy of these radiometers is unknown during this period. Thus, if the two diffuse shortwave measurements agree to within $8 \mathrm{Wm}^{-2}$ or $5 \%$, the two values 
are averaged for the best estimate. If the two measurements do not agree to this level, then a highly likely cause is the demonstrated sampling difference effect of Figures 1 and 2. In this case, the 1-second sample data is used if available, else the 20 -second sample data.

For the downwelling longwave irradiance, again the actual calibration accuracy of these radiometers is unknown during this period. We apply the same strategy as for the diffuse shortwave irradiance. If the two-downwelling longwave measurements agree to within $5 \mathrm{Wm}^{-2}$ or $1 \%$, the two values are averaged for the best estimate. If the two measurements do not agree to this level, then a highly likely cause is the demonstrated sampling difference effect of Figures 1 and 2 . In this case, the 1-second sample data is used if available, else the 20-second sample data.

The total downwelling shortwave irradiance is calculated as the sum of the direct and diffuse components, if available. If the direct and/or diffuse shortwave is not available, then by definition the downwelling shortwave measured by the unshaded pyranometers is the best available. In this case, the 1-second sampled data are used if available, else the 20-second sampled data.

The broadband shortwave surface albedo is calculated as the ratio of the upwelling shortwave irradiance over the downwelling shortwave irradiance, if both are available and non-negative, for daylight hours including civil twilight (solar zenith angle $<93^{\circ}$ ).

The Net radiative surface flux is calculated as the sum of all upwelling and downwelling components when all are available. During daylight (solar zenith angle $<93^{\circ}$ ), this is calculated as the (downwelling minus the upwelling shortwave irradiance), plus the (downwelling minus the upwelling longwave irradiance). For nighttime, only the longwave components are used.

\section{Best Estimate Flux Data File Format and QC Flags}

The ARM Best Estimate Flux VAP is in the late stages of beta testing. Obviously, the VAP is producing quality output; else the files could not be included on these CDs. If these files are not available from the ARM Archive through the User Interface by the time of the 2002 ARM Science Team Meeting, then they will be so shortly after. The files are named per the following example:

sgpbeflux1longC1.c1.20011231.000000.cdf

The Manual Best Estimate Flux files contained on these CDs will be made available through the ARM External Data Archive, since they have not been produced by an ARM automated code and are not able to be automatically reprocessed. Should anyone find problems with the Manual Best Estimate data, please contact me (Chuck Long) so that I may address the concerns, and reprocess and resubmit the files to the External Data Archive if needed.

Both the ARM VAP and Manually produced Best Estimate products contain the same information and values, and have the same formats. The files include data QC flags, and where possible the difference between the two data. For the Best Estimate VAP, the difference listed is between the two values used in that average. For the Manual Best Estimate, these represent the 
difference in the two measurements whether both went into the best estimate value or not. In addition, while the data from the ARM Archive is only produced in NetCDF format, these CDs contain both NetCDF and ASCII versions of the output files. Table 1 lists the data labels for both the NetCDF and ASCII versions, and brief descriptions of each. The various values of the included data QC flags, and a brief description of meaning, is as follows:

For the direct normal and diffuse shortwave, and downwelling longwave:

Flag $=2 \mathrm{E} 13+\mathrm{C} 1$

Flag $=1$ BRS $+\mathrm{C} 1$

Flag $=0$ BRS + E13

Flag $=-1$ BRS only

Flag $=-2$ E13 only

Flag $=-3$ C1 only

Flag $=4$ Not enough info.

Flag $=-4$ All instruments are down

For the upwelling shortwave and longwave:

Flag $=0 \mathrm{E} 13+\mathrm{C} 1$

Flag $=1$ E13 only

Flag $=2$ C1 only

Flag $=4$ Not enough info.

Flag $=-4$ All instruments are down

Table 1. NetCDF and ASCII Data Labels for the Best Estimate Flux Files

\begin{tabular}{|c|c|c|c|}
\hline NetCDF & ASClI & Comment & Units \\
\hline down_short_hemisp & SWd & total (global) downwelling shortwave irradiance & $\mathrm{Wm}^{-2}$ \\
\hline dow_long_hemisp & IRd & downwelling lonchave irradiance & $\mathrm{Wm}^{-2}$ \\
\hline short_direct_nomal & DirN & direct nornal shortwave irradiance & $\mathrm{Wm}^{-2}$ \\
\hline down_short_diffuse_hemisp & Dif & downwelling diffuse shortwave irradiance & $\mathrm{Wm}^{-2}$ \\
\hline up_short_hemisp & SWu & upwelling shortwave irradiance & $\mathrm{Nm}^{-2}$ \\
\hline up_long_hemsp & IRu & upwelling longwcve irradiance & $\mathrm{Wm}^{-2}$ \\
\hline net_surface_radiation & Net & net surface radiative flux & $\mathrm{Wm}^{-2}$ \\
\hline albedo & Alb & broadband shortivaw surface albedo & none \\
\hline zenith & Zen & solar zenith angle & degrees \\
\hline down_long_hemisp_flag & IRdflg & QC flag - IRd & none \\
\hline short_direct_nomal_flag & Dirflg & QC flag - DirN & none \\
\hline down_short_diffuse_hemisp_flag & Difflg & QC flag - Dif & none \\
\hline up_short_hemisp_flag & SWuilg & QC flag - SWu & none \\
\hline up_long_hemsp_flag & IRuflg & QC flag - IRu & none \\
\hline short_direct_nomal_diff & Clirdiff & difference of the two values - Cirtv & $\mathrm{Wm}^{-2}$ \\
\hline down_short_diffuse_hemisp_diff & Difdiff & difference of the two values - Dif & $\mathrm{Wm}^{-2}$ \\
\hline down_long_hemisp_diff & IRddif & difference of the tho values - IRd & $\mathrm{Wm}^{2}$ \\
\hline up_short_hemisp_diff & SWudiff & difference of the two values - SWu & $\mathrm{Wm}^{-2}$ \\
\hline up_long_hemsp_diff & IRudiff & difference of the two values - IRu & $\mathrm{Wm}^{-2}$ \\
\hline
\end{tabular}




\section{SW Flux Analysis from Best Estimate Flux}

The Shortwave Flux Analysis files currently in the ARM Archive do not contain corrected diffuse shortwave measurements. The Diffuse Correction VAP output files only recently became publicly available, and only for the SIRS type of radiometer systems data. The Diffuse Correction VAP is just now undergoing beta testing for processing the older SIROS format data. In addition, we are working on updating the Shortwave Flux Analysis VAP to use the Diffuse Correction VAP as input, as well as the Best Estimate Flux VAP for the SGP CF site. This work is occurring along with a recent adaptation of the SW Flux Analysis algorithm to be able to process data from sites that do not experience individual days that are "clear enough" for daily fitting, such as the ARM tropical western Pacific sites (for a definition of “clear enough," see Long and Ackerman 2000). (More information on the adaptation to the TWP climate regime for the SW Flux Analysis VAP is presented in the ARM STM 2002 poster "An Evaluation of Cloud Cover, Cloud Effect, and Surface Radiation Budgets at the SGP and TWP ARM Sites” by Gaustad and Long.) These improvements and upgraded Shortwave Flux Analysis files will be available from the ARM Archive in the near future. In the mean time, I have processed the Best Estimate Flux data contained on these CDs through the code, and have included the 15-minute ASCII version of the output.

The Shortwave Flux Analysis file names follow the convention:

YYYYMMDD.c15

Where YYYY is the year, MM is the month of the year, and DD is the day of the month. The data inside are in columns and rows, the rows corresponding to data at a given time stamp, the time stamp representing the BEGINNING of the averaging period. The columns are the variable values. Each column has an ASCII text header for the variable as per the following excerpt from Long (2001):

Coefficient Data:

$\begin{array}{ll}\text { Label } & \text { Meaning } \\ \text { Date } & \text { date in YYYYMMDD format, based on LST } \\ \text { DFRa } & \text { "a” coefficient calculated for diffuse ratio } \\ \text { DFRb } & \text { "b” coefficient calculated for diffuse ratio } \\ \text { CSWa } & \text { “a” coefficient calculated for clear-sky global SW } \\ \text { CSWb } & \text { "b” coefficient calculated for clear-sky global SW } \\ \text { CSSWa } & \text { "a” coefficient calculated for clear-sky sum SW } \\ \text { CSSWb } & \text { "b” coefficient calculated for clear-sky sum SW } \\ \text { SCORc } & \text { offset coefficient calculated for clear-sky global-sum difference } \\ \text { SCORa } & \text { "a” coefficient calculated for clear-sky global-sum difference } \\ \text { SCORb } & \text { "b” coefficient calculated for clear-sky global-sum difference } \\ \text { ALBa/UVBa } & \text { "a” coefficient calculated for clear-sky albedo/UV } \\ \text { ALBb/UVBb } & \text { "b” coefficient calculated for clear-sky albedo/UV } \\ \text { AvgAU } & \text { daily average earth-sun distance in AUs }\end{array}$


NOTE: If "Date" is all zeros, then the code was run in "one fit for all data" mode. In this case, to produce the daily clear-sky CSW and CSSW, divide the "a" coefficient by the square of the “AvgAU”.

Irradiance Data:

\begin{tabular}{|c|c|}
\hline Label & Meaning \\
\hline Zdate & date in YYYYMMDD format, based on GMT \\
\hline Ztim & time in hhmm format, based on GMT \\
\hline Ldate & date in YYYYMMDD format, based on LST \\
\hline Ltim & time in hhmm format, based on LST \\
\hline difcld & Normalized Diffuse Cloud Effect used for sky cover estimation \\
\hline CFstdev & Standard deviation from mean of sky cover \\
\hline cf & 15-minute average sky cover \\
\hline Ncf & Number of 1-minute data available for 15-minute avg. sky cover \\
\hline Ncsw & Number of 1-minute data available for 15-minute avgs. \\
\hline Nssw & Number of 1-minute data available for 15-minute avg. SSW \\
\hline Nclr & Number of 1-minute data detected as clear in 15-minute avg. \\
\hline CosZ & Cosine of the solar zenith angle (used for fitting) \\
\hline AU & Earth - Sun distance in AUs \\
\hline tsw & measured downwelling SW from global pyranometer $\left(\mathrm{W} / \mathrm{m}^{\wedge} 2\right)$ \\
\hline csw & clear-sky downwelling SW from global pyranometer $\left(\mathrm{W} / \mathrm{m}^{\wedge} 2\right)$ \\
\hline tswfcg & csw minus tsw $\left(\mathrm{W} / \mathrm{m}^{\wedge} 2\right)$ \\
\hline dif & measured downwelling diffuse $\mathrm{SW}\left(\mathrm{W} / \mathrm{m}^{\wedge} 2\right)$ \\
\hline cdif & clear-sky downwelling diffuse SW (W/m^^2) \\
\hline diffcg & cdif minus dif $\left(\mathrm{W} / \mathrm{m}^{\wedge} 2\right)$ \\
\hline $\operatorname{dir}$ & measured downwelling direct $\mathrm{SW}\left(\mathrm{W} / \mathrm{m}^{\wedge} 2\right)$ \\
\hline cdir & clear-sky downwelling direct SW (W/m^2) \\
\hline difr & measured diffuse ratio $($ dif/tsw $)\left(\mathrm{W} / \mathrm{m}^{\wedge} 2\right)$ \\
\hline cdifr & clear-sky diffyse ratio (cdif/csw) $(\mathrm{W} / \mathrm{m} \wedge 2)$ \\
\hline ssw & measured downwelling sum (dif+dir) SW (W/m^2) \\
\hline cssw & clear-sky downwelling sum (cdif+cdir) SW (W/m^^2) \\
\hline sswfcg & CSsw minus ssw \\
\hline alb/UVB & measured albedo (no units) or UVB (units as in input data) \\
\hline calb/CUVB & clear-sky albedo (no units) or UVB (units as in input data) \\
\hline IRdn & measured downwelling longwave \\
\hline IRup & measured upwelling longwave \\
\hline Net & total net surface radiative flux \\
\hline Drdif & difference between measured values of 2 direct normal instruments \\
\hline Dfdif & difference between measured values of 2 diffuse instruments \\
\hline Iddif & difference between measured values of 2 downwelling LW instrumen \\
\hline Sudif & difference between measured values of 2 upwelling SW instruments \\
\hline Iudif & difference between measured values of 2 upwelling LW instruments \\
\hline
\end{tabular}




\section{Acknowledgements}

First and foremost, I would like to thank those who were responsible for the existence of the radiometer measurements upon which this work is based. These include the SGP Site Operations and Scientist Office, the Calibration Facility, and the ARM Data Quality Manager personnel. Quite often the dedicated effort of these people in the daily monitoring and maintenance of the radiometer systems is overlooked, under appreciated, and unacknowledged in our publications of results that use these data.

There have been many others whose effort and expertise has made this work possible. I thank Marv Wesely for his information on the history of problems encountered with the radiometer systems that went into the manually produced product, as well as further information provided by Ted Cress, Tom Stoffel, and Robin Perez. I thank Katarina Younkin, Yan Shi, Tim Shippert, and Krista Gaustad for their efforts and expertise in producing the various ARM VAPs whose outputs were used here. The efforts of these folks also often go unrecognized.

In the larger sense, all scientific contributions related to understanding these types of measurements and instruments had a part. A few names directly related to this project such as Ells Dutton, Joe Michalsky, Mike Rubes, John DeLuisi, and all the BSRN folks come to mind. If I have missed anyone, I apologize. I have tried to use what I feel are justifiable decisions using substantiated information to make the choices on what is "best." I hope you agree with my reasoning, and find these data useful. For all I have listed, and those I haven't, the good that is presented here is because of them. Any errors, misstatements, or inconsistencies contained here are mine and mine alone.

\section{Corresponding Author}

Dr. Charles N. Long

Senior Research Scientist

Atmospheric Radiation Measurement Program

Pacific Northwest National Laboratory

P. O. 999, MSIN: K9-24

Richland, WA, USA 99352

Ofc.: 1 (509) 372-4917

Fax: 1 (509) 372-6168

e-mail: chuck.long@pnl.gov

\section{References}

Dutton, E. G., J. J. Michalsky, T. Stoffel, B. W. Forgan, J. Hickey, D. W. Nelson, T. L. Alberta, and I. Reda, 2001: Measurement of Broadband Diffuse Solar Irradiance Using Current Commercial Instrumentation with a Correction for Thermal Offset Errors. J. of Atmospheric and Oceanic Technology, Vol. 18, March 2001 
Gaustad, K., and C. N. Long, 2002: An evaluation of cloud cover, cloud effect, and surface radiation budgets at the SGP and TWP ARM Sites. In Proceedings of the Twelfth Atmospheric Radiation Measurement (ARM) Science Team Meeting, St. Petersburg, Florida, April 8-12, 2002.

Long, C. N., 2001: The Shortwave (SW) Clear-Sky Detection and Fitting Algorithm: Algorithm Operational Details and Explanations, Atmospheric Radiation Measurement Program Technical Report, ARM TR-004. Available URL: http://www.arm.gov/docs/documents/tech_reports/index.html

Long, C. N., K. Younkin, and D. M. Powell, 2001: Analysis of the Dutton et al. IR Loss Correction Technique Applied to ARM Diffuse SW Measurements. In Proceedings of the Eleventh Atmospheric Radiation Measurement (ARM) Science Team Meeting. Available URL:

Long, C. N. and T. P. Ackerman, 2000: Identification of Clear Skies from Broadband Pyranometer Measurements and Calculation of Downwelling Shortwave Cloud Effects. J. of Geophys. Research, 105, No. D12, 15609-15626.

Long, C. N., 1996: Report on Broadband Solar Radiometer Inconsistencies at the Atmospheric Radiation Measurement (ARM) Southern Great Plains (SGP) Central Facility During the ARM Enhanced Shortwave Experiment (ARESE), Atmospheric Radiation Measurement Program Technical Report, ARM TR-003. Available URL: http://www.arm.gov/docs/documents/tech_reports/index.html

Michalsky, J., E. Dutton, M. Rubes, D. Nelson, T. Stoffel, M. Wesely, M. Splitt, and J. DeLuisi, 1999: Optimal Measurement of Surface Shortwave Irradiance Using Current Instrumentation. J. of Atmos. and Ocean. Tech., 16, pp. 55-69.

Ohmura A., E. G. Dutton, B. Forgan, C. Frohlich, H. Gilgen, H. Hegner, A. Heimo, G. KonigLanglo, B. McArthur, G. Muller, R. Phillipona, R. Pinker, C. H. Whitlock, K. Dehne, and M. Wild, 1998: Baseline Surface Radiation Network (BSRN/WCRP): New Precision Radiometry for Climate Research. Bull. Of the Amer. Meteo. Soc., 79, No, 10, pp. 2115-2136.

Shi, Y., and C. N. Long, 2002: Techniques and methods used to determine the best estimate of radiation fluxes at SGP Central Facility. In Proceedings of the Twelfth Atmospheric Radiation Measurement (ARM) Science Team Meeting, St. Petersburg, Florida, April 8-12, 2002.

Younkin, K., and C. N. Long, 2002: Results of the Dutton at al. IR loss correction VAP: Statistical analysis of corrected and uncorrected SW measurements. In Proceedings of the Twelfth Atmospheric Radiation Measurement (ARM) Science Team Meeting, St. Petersburg, Florida, April 8-12, 2002. 